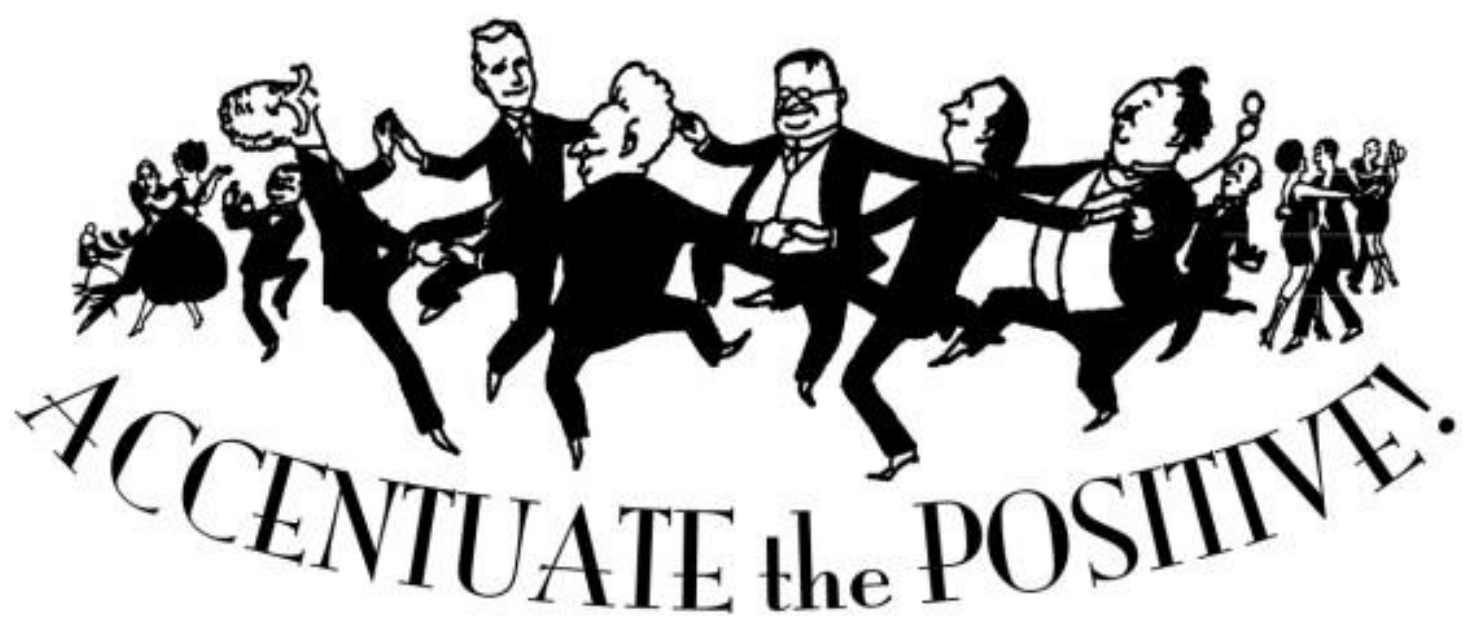

2012 Charleston Conference

32nd Annual Conference on Issues in Book and Serial Acquisitions

Point of Care Tools and Libraries

(the 12th Annual Health Sciences Lively Lunch)

Friday, November 9, 2012

Point of Care Decision Tools: An Environmental Scan

Presenter: Susan Klimley, Health Sciences Library, Columbia University

A sampling of references used for research and referred to during the presentation:

\title{
JOURNAL ARTICLES
}

Informed Consent Beyond the Physician-Patient Encounter: Tort Law Implications of ExtraClinical Decision Support Tools

Sawicki, Nadia N.

21 ANNALS HEALTH L. 1 (2012).

(Accessible, Creative Commons license, http://ecommons.luc.edu/law facpubs/161/)

Beyond "Safe and Effective": The Role of the federal government in supporting and disseminating comparative-effectiveness research

Francis, $\mathrm{MH}$

Ann Health Law 2012 21:2 329-82 PMID: 22606919

Speed of updating online evidence based point of care summaries: prospective cohort analysis Banzi R, Cinquini M, Liberati A, Moschetti I, Pecoraro V, Tagliabue L, Moja L.

BMJ. 2011 Sep 23;343:d5856. PMID: 21948588

Making evidence-based medicine doable in everyday practice White B.

Fam Pract Manag. 2004 Feb;11(2):51-8. PMID: 15011482 
Bringing diagnosis into the quality and safety equations

Graber ML, Wachter RM, Cassel CK.

RTI International, 1 Breezy Hollow, St James, NY 11780, USA. mgraber@rti.org

JAMA. 2012 Sep 26;308(12):1211-2. PMID: 2301170

The quality, breadth, and timeliness of content updating vary substantially for 10 online medical texts: an analytic survey

Prorok JC, Iserman EC, Wilczynski NL, Haynes RB.

Health Informat Res Unit, Dept of Clinical Epidemiology and Biostatistics, McMaster University

J Clin Epidemiol. 2012 Sep 10. pii: S0895-4356(12)139-4. PMID: 22974495

Factors associated with medical knowledge acquisition during internal medicine residency McDonald FS, Zeger SL, Kolars JC.

General Internal Medicine-Hospital Internal Medicine, Mayo Clinic College of Medicine, 200 1st Street SW, Rochester, MN 55905, USA. mcdonald.furman@mayo.edu

J Gen Intern Med. 2007 Jul;22(7):962-8. Epub 2007 Apr 28.

PMID: 17468889; PMCID: PMC2219722

Use of UpToDate and outcomes in US hospitals

Isaac T, Zheng J, Jha A.

Division of General Internal Medicine and Primary Care, Beth Israel Deaconess Medical Center, Boston, MA, USA.

J Hosp Med. 2012 Feb;7(2):85-90. PMID: 22095750

Developing and using a rubric for evaluating evidence-based medicine point-of-care tools Shurtz S, Foster MJ.

Instructional Service Librarian, Medical Sciences Library, Texas A\&M University, 4462 TAMU

College Station, TX 77843-4462, USA.sshurtz@library.tamu.edu

J Med Libr Assoc. 2011 Jul;99(3):247-5. PMID: 21753917; PMCID: PMC3133902

Too many alerts, too much liability: Sorting through the malpractice implications of drug-drug interaction clinical decision support

Ridgely, SM and Greenberg, MD.

Saint Louis University Journal of Health Law \& Policy, 2012, 5:2 257-296

POSTER

Visual informatics: real-time visual decision support

Papier A, Allen E, McDermott M.

Poster presented at: American Medical Informatics Association 2001 Annual Symposium;

November 3-7, 2001; Washington, DC.

Viewed Nov. 2012: http://www.visualdx.com/file download/51

WEB REPORT

Point of Care decision-making tools-Overview HLWIKI Canada

Viewed Nov. 2012:

http://hlwiki.slais.ubc.ca/index.php/Point of care decision-making tools - Overview 
